\title{
The use of a tailored surgical technique for minimally invasive esophagectomy
}

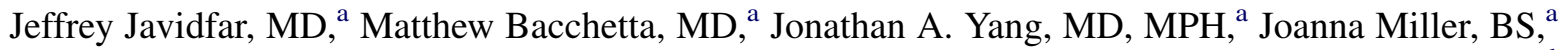 \\ Frank D'Ovidio, MD, PhD, ${ }^{\mathrm{a}}$ Mark E. Ginsburg, MD, ${ }^{\mathrm{a}}$ Lyall A. Gorenstein, MD, ${ }^{\mathrm{a}}$ Marc Bessler, MD, ${ }^{\mathrm{b}}$ and \\ Joshua R. Sonett, MD
}

\begin{abstract}
Objective: Uncertainty exists among surgeons as to whether minimally invasive esophagectomy (MIE) is a comparable operation to open esophagectomy (OE). The surgical technique and oncologic dissection should not be degraded when using a minimally invasive approach.
\end{abstract}

\begin{abstract}
Methods: We reviewed a single hospital's experience with both OE and MIE. From 2000 to 2010, 257 patients underwent esophagectomy by 1 of 3 surgical techniques: transhiatal, Ivor Lewis, or 3-hole.
\end{abstract}

\begin{abstract}
Results: Of the 257 patients (median age, 67 years; range, 58-74), 92 underwent MIE. Both groups were comparable in terms of gender, age, comorbidities, surgical technique, and induction chemotherapy and radiotherapy. The overall median follow-up was 29.5 months (range, 9.9-61.5). The MIE group had a significantly shorter operative time (MIE vs OE, 330 vs 365 minutes, $P=.04$ ), length of stay (MIE vs OE, 9 vs 12 days, $P<.01$ ), intensive care unit admission rate (MIE vs OE, $55 \%$ vs $81 \%, P<.01$ ), intensive care unit length of stay (MIE vs OE, 1 vs 2 days, $P<.01$ ), and estimated blood loss (MIE vs OE, 100 vs $400 \mathrm{~mL}, P<.01$ ). More lymph nodes were harvested in the MIE group than in the OE group (17 vs 11 nodes, $P<.01)$. There were insignificant differences in 30-day mortality (MIE vs OE, $2.2 \%$ vs $3.0 \% ; P=.93$ ) and overall survival $(P=.19$ ), as well as in the rates of all complications, except pneumonia (MIE vs OE, $2 \%$ vs $13 \% ; P=.01$ ).
\end{abstract}

Conclusions: A thoracic surgeon can safely tailor the MIE to a patient's anatomy and oncologic demands while maintaining equivalent survival. (J Thorac Cardiovasc Surg 2012;143:1125-9)

The surgical treatment of esophageal cancer involves esophagectomy with en bloc nodal dissection and reconstitution of the alimentary track. There are 3 standard techniques for performing an open esophagectomy (OE): the transhiatal esophagectomy (THE), transthoracic or Ivor Lewis (IL) esophagectomy, and McKeown's 3-phase or 3-hole (3H) technique. ${ }^{1-3}$ In the years since, both prospective and retrospective studies have found no statistically significant advantage for 1 technique over another, as it pertains to OE. ${ }^{4-6}$ Each technique has its risks and benefits. ${ }^{7}$ Accordingly, the characteristics and location of the esophageal tumor, along with the patient's global health, can contribute to determining which procedure is performed. ${ }^{4}$

The traditional OE entails significant morbidity. ${ }^{5}$ The need to reduce this morbidity has led to the development of minimally invasive techniques for performing each of the 3 types of esophagectomy. ${ }^{8,9}$ Owing to the technically

\footnotetext{
From the Divisions of Cardiothoracic Surgery a and Minimally Invasive Surgery, ${ }^{\mathrm{b}}$ Columbia University Medical Center, New York, NY.

Disclosures: Authors have nothing to disclose with regard to commercial support.

Read at the 37th Annual Meeting of The Western Thoracic Surgical Association,

Colorado Springs, Colorado, June 22-25, 2011.

Received for publication June 20, 2011; revisions received Dec 10, 2011; accepted for publication Jan 25, 2012.

Address for reprints: Joshua R. Sonett, MD, Division of Cardiothoracic Surgery, Columbia University Medical Center, 161 Fort Washington Avenue, Herbert Irving Pavilion, Suite 301, New York, NY, 10032 (E-mail: JS2106@ columbia.edu). $0022-5223 / \$ 36.00$

Copyright (c) 2012 by The American Association for Thoracic Surgery doi:10.1016/j.jtcvs.2012.01.071
}

demanding nature of this procedure, access to minimally invasive esophagectomy (MIE) has been limited to select specialized centers that have been able to show comparable results. However, such high-volume centers tend to advocate a specific minimally invasive technique, such as Ivor Lewis or transhiatal esophagectomy, reflecting the learning curve and experience of each institution. For the MIE to be truly equivalent in the treatment of esophageal cancer to OE, patients would need to be offered the technique best suited to them and their disease.

The outcomes of patients with esophageal carcinoma who were treated with a tailored OE were compared with those of patients treated with a tailored MIE. We examined whether a difference between the outcomes in these 2 cohorts existed. A unique aspect of our study population was that disease characteristics dictated which minimally invasive technique they were offered.

\section{METHODS}

The present study, which was approved by the Columbia University Medical Center institutional review board, was a retrospective review of a single institution's experience with both OE and MIE.

\section{Patient Selection}

From February 2000 to June 2010, 257 consecutive patients underwent esophagectomy for a preoperative diagnosis of cancer using 1 of 3 surgical techniques: THE, IL, or $3 \mathrm{H}$.

Descriptive statistics were collected. A thorough review of the inpatient and outpatient clinical records was conducted to ascertain information 


$$
\begin{aligned}
& \text { Abbreviations and Acronyms } \\
& \begin{array}{l}
3 \mathrm{H}=3 \text {-hole } \\
\mathrm{MIE}=\text { minimally invasive esophagectomy } \\
\mathrm{OE}=\text { open esophagectomy } \\
\mathrm{THE}=\text { transhiatal esophagectomy }
\end{array}
\end{aligned}
$$

regarding the patient's operative course, postoperative hospitalization, possible intensive care unit (ICU) stay, and pathology reports. Staging was determined using the American Joint Committee on Cancer Staging Manual, 7 th edition. ${ }^{10}$ The short- and long-term follow-up data, including complication rates or adverse events, were also documented. Postoperative pneumonia was defined by a new infiltrate on chest $\mathrm{x}$-ray plus 2 of the following: leukocytosis, hypoxia, fever, or positive sputum culture. ${ }^{11}$ Owing to the limited availability of the retrospective data, the "operative time" was defined as the interval from when the patient was anesthesia ready to when the patient left the operating room. The patients were followed up routinely by their primary surgeon and by the gastroenterologists and oncologists within the same healthcare system.

The demographic characteristics of the study population were recorded (Table 1). A total of 7 surgeons performed the open and minimally invasive procedures.

\section{Surgery}

The open procedures were conducted in standard fashion, specific to each technique. ${ }^{12}$ The minimally invasive procedures were completed using slight modifications of the described techniques for minimally invasive IL esophagectomy, THE, and 3H esophagectomy. ${ }^{4,13-15}$ All esophagectomies received a feeding jejunosotomy tube. To facilitate gastric emptying, the open procedures included pyloroplasty. The minimally invasive procedures initially also included pyloroplasty that was later transitioned to endoscopically injected botulinum toxin into the pylorus combined with dilation. To expedite work in the foregut, the MIE was performed on a bariatric surgery table that could tilt $45^{\circ}$, and long laparoscopic and thoracoscopic instruments were used. A highdefinition camera and monitors allowed for improved visualization, and a video mediastinoscope facilitated cervical neck dissection.

Hybrid procedures, such as laparoscopic gastric mobilization followed by thoracotomy or laparotomy with thoracoscopic mobilization, were not included in the analysis. A distinction was made between hybrid procedures $(\mathrm{n}=8)$ and conversion to $\mathrm{OE}(\mathrm{n}=3)$. In the latter, a procedure that was intended to be performed entirely by a minimally invasive approach required open completion.

The lymphadenectomy performed was standard and included upper and lower mediastinal paraesophageal, pericardial, celiac trunk, lesser curvature of the stomach, left gastric artery, splenic artery, and carinal nodal basins. ${ }^{16}$ In general, induction therapy was offered to patients who had T3 or N1 disease by endoscopic ultrasonography and who were also thought to have performance status to support trimodality therapy. The chemotherapy given was a mix of differing regimens, as the referring oncologists most often provided the therapy. Most were cisplatin-based regimens. Radiation was standard dosing to $50.5 \mathrm{~Gy}$, as tolerated.

\section{Statistical Analysis}

All statistical analyses were performed with a statistical software package (StataCorp, College Station, Tex). The values are reported as the median and interquartile range. To minimize potential concerns regarding the normality of the data distribution, nonparametric rank sum tests were used to compare the continuous variables, as appropriate. Categorical variables were compared using chi-square tests. The mortality data were confirmed using the Social Security Death Index. The follow-up time was defined as the postoperative time to the last office visit. Survival comparisons were made with Kaplan-Meier analysis, with survival estimates compared using a log-rank test. Overall survival was defined as the time from the date of surgery to death or the last follow-up visit through July 15, 2011.

\section{RESULTS}

From February 2000 to June 2010, 257 patients underwent esophagectomy at our institution. During this period, 92 MIEs were performed. Of the 257 patients, 194 were men $(75 \%)$, and the median patient age was 67 years (range, 58-74). The OE and MIE cohorts were equally matched in terms of gender, age, and instances of induction chemotherapy and radiation (Table 1). The 3 surgical techniques (THE, $\mathrm{IL}, 3 \mathrm{H}$ ) were distributed similarly between the $\mathrm{OE}$ and MIE $(P=.27)$. A significant difference was found in the pathologic tumor stage between the 2 groups $(P=.01)$. This was attributed to more stage III disease in the OE cohort ( $42 \%$ vs $29 \%$ in the MIE cohort; Table 1). All the patients with stage 0 disease on the final pathologic examination (10 MIE patients and 4 OE patients) had preoperative clinical stage I (6 MIE and $2 \mathrm{OE}$ ), II (2 MIE and $1 \mathrm{OE}$ ), and III (2 MIE and $1 \mathrm{OE}$ ) according to the endoscopic ultrasound findings. The patients with stage II and III disease underwent combined induction chemoradiotherapy and were believed to have achieved a complete pathologic response.

The overall median follow up was 29.5 months (range, 9.9-61.5). The median follow-up for the OE group was longer (52 months; range, 13-83) than that for the MIE group (21 months; range, 11-37). The final surgical pathologic examination revealed that $91 \%$ of the MIE group had adenocarcinoma compared with $84 \%$ of the OE group $(P=.14)$. $\mathrm{R} 0$ or complete pathologic resection was accomplished in $99 \%$ of the MIE cohort compared with $94 \%$ of the OE group $(P=.10)$. The lone MIE patient had radial margins involved and postoperatively was found to have stage IIIc disease on final pathologic examination. All the patients without R0 resection in the OE group had positive radial margins on final pathologic examination. The cohort that received induction chemotherapy and radiotherapy represented $55 \%(\mathrm{n}=51)$ of the MIE patients and 58\% $(\mathrm{n}=96)$ of the OE patients $(P=.29)$.

When comparing the MIE and OE group, the MIE cohort had a significantly shorter operative time, length of stay, ICU admission rate, ICU length of stay, and estimated blood loss (Table 2). Significantly more lymph nodes were harvested in the MIE group (Table 2). Subgroup analysis demonstrated significantly improved nodal dissection among the MIE performed using the IL and THE techniques and an insignificant trend favoring MIE among the $3 \mathrm{H}$ subgroup. The median number of lymph nodes harvested per final pathologic stage was greater for the MIE approach $(P<.01)$.

With the exception of postoperative pneumonia, there was not a significant difference between the rates of adverse events, including anastomotic or chyle leak requiring reoperation, strictures needing dilation, recurrent laryngeal 
TABLE 1. Baseline characteristics

\begin{tabular}{|c|c|c|c|}
\hline Characteristic & MIE & OE & $P$ value \\
\hline Sample size (n) & $92(36)$ & $165(64)$ & \\
\hline Age (y) & & & .74 \\
\hline Mean & 65 & 68 & \\
\hline Range & $56-74$ & $60-74$ & \\
\hline Men (n) & $71(77)$ & $122(74)$ & .99 \\
\hline Adenocarcinoma & $84(91)$ & $139(84)$ & .14 \\
\hline Squamous cell carcinoma & $8(9)$ & $26(16)$ & .14 \\
\hline R0 resection & $91(99)$ & $155(94)$ & .10 \\
\hline Esophagectomy technique & & & .81 \\
\hline Transhiatal & $49(53.3)$ & $81(49)$ & \\
\hline Ivor Lewis & $27(29.3)$ & $53(32)$ & \\
\hline 3-Hole & $16(17.3)$ & $31(19)$ & \\
\hline Pathologic tumor stage & & & .01 \\
\hline 0 & $10(11)$ & $4(2)$ & \\
\hline I & $36(39)$ & $65(40)$ & \\
\hline II & $19(21)$ & $27(16)$ & \\
\hline III & $27(29)$ & $69(42)$ & \\
\hline Surgical technique & & & .29 \\
\hline Transhiatal & $27(53)$ & $47(49)$ & \\
\hline Ivor Lewis & $15(29)$ & $31(32)$ & \\
\hline 3-Hole & $9(18)$ & $18(19)$ & \\
\hline \multicolumn{4}{|l|}{ Preoperative comorbidity } \\
\hline Hypertension & $45(49)$ & $84(51)$ & .68 \\
\hline Diabetes & $22(24)$ & $35(21)$ & .53 \\
\hline Hyperlipidemia & $26(28)$ & $31(19)$ & .11 \\
\hline $\begin{array}{l}\text { Chronic obstructive pulmonary } \\
\text { disease }\end{array}$ & $9(10)$ & $23(14)$ & .26 \\
\hline Asthma & $9(10)$ & $12(7)$ & .46 \\
\hline Coronary artery disease & $9(9)$ & $23(14)$ & .26 \\
\hline
\end{tabular}

Data presented as mean and range or $\mathrm{n}(\%)$. MIE, Minimally invasive esophagectomy; $O E$, open esophagectomy.

nerve injury, and conduit torsion or bleeding requiring intervention (Table 3). The cases of conduit torsion were limited to the THE and $3 \mathrm{H}$ groups. Similarly, the incidence of recurrent laryngeal nerve injury was associated with the presence of cervical incision and anastomosis (THE and $3 \mathrm{H}$ ), and no significant difference was found between the MIE and $\mathrm{OE}$ cohorts $(P=.21)$. Among the 3 MIE techniques, the data suggest that there was in an insignificant difference in the number of nodes harvested $(P=.42)$, the incidence of complications $(P=.65)$, and the survival rates $(P=.9)$.

After stratifying for tumor stage and surgical technique, there was an insignificant statistical difference in the 30day mortality rate (MIE, $2.2 \%$ vs $\mathrm{OE}, 3.0 \% ; P=.93$ ), in-hospital mortality rate (MIE, $3.2 \%$ vs OE, $4.2 \%$; $P=1.00)$, and the overall survival rate $(P=.19$; Figure 1). Similarly, no significant survival difference was found between the 2 approaches in the cohort that received neoadjuvant chemotherapy $(P=.25)$.

\section{CONCLUSIONS}

The present series reflects a single institution's initial experience with transitioning from OE to MIE. It was our
TABLE 2. Results

\begin{tabular}{|c|c|c|c|}
\hline Variable & MIE & OE & $P$ value \\
\hline \multicolumn{4}{|l|}{ Operative time* $(\min )$} \\
\hline Median & 330 & 365 & .04 \\
\hline Interquartile range & $288-411$ & $319-472$ & \\
\hline Length of stay (d) & & & $<.01$ \\
\hline Median & 9 & 12 & \\
\hline Interquartile range & $8-14$ & $10-21$ & \\
\hline ICU admission rate $(\%)$ & 55 & 81 & $<.01$ \\
\hline ICU length of stay (d) & & & $<.01$ \\
\hline Median & 1 & 2 & \\
\hline Interquartile range & $0-2$ & $1-4$ & \\
\hline Estimated blood loss (mL) & & & $<.01$ \\
\hline Median & 100 & 400 & \\
\hline Interquartile range & $50-300$ & $250-600$ & \\
\hline \multicolumn{4}{|l|}{ Nodes harvested } \\
\hline Overall & & & $<.01$ \\
\hline Median & 17 & 11 & \\
\hline Interquartile range & $12.5-24.5$ & $7-16$ & \\
\hline \multicolumn{4}{|l|}{ Transhiatal } \\
\hline Median & 17 & 13 & $<.01$ \\
\hline Interquartile range & $14-22.5$ & $5-15$ & \\
\hline \multicolumn{4}{|l|}{ Ivor Lewis } \\
\hline Median & 19 & 12 & .03 \\
\hline Interquartile range & $13-27$ & $9-19$ & \\
\hline \multicolumn{4}{|l|}{ Three-hole } \\
\hline Median & 16 & 10 & .39 \\
\hline Interquartile range & $12-23$ & $11-19$ & \\
\hline Conversion to $\mathrm{OE}$ & $3(3)$ & NA & NA \\
\hline
\end{tabular}

Data in parentheses are percentages. MIE, Minimally invasive esophagectomy; $O E$, open esophagectomy; $I C U$, intensive care unit. *From anesthesia ready to out of the operating room.

intent to offer identical surgical approaches according to the patient and tumor characteristics. Two similar cohorts undergoing either OE or MIE were studied and found to have comparable survival. Using the same surgical decision making as for OE, a specific MIE technique (THE, IL, or $3 \mathrm{H})$ was tailored to the patient's needs and was not limited by institutional preference or experience. This differed from previous studies in which often only 1 minimally invasive technique was offered to patients. ${ }^{15,17}$ Mostly, patients with early-stage or limited gastroesophageal junction disease were transhiatal candidates and those with more advanced and/or bulkier disease underwent either an IL or $3 \mathrm{H}$ esophagectomy. This led to a correlation between surgical technique and pathologic tumor stage.

The present study included the work of 7 surgeons. Initially, the MIEs were performed by 2 surgeons and the experience was extended through the group. The number of surgeons involved counters the single-institution nature of the study and might make the data more applicable. No operator-specific preferences were present in the surgical approach or esophagectomy technique used, instead the patient, nature of the disease, and the anatomy determined the approach and technique used. Furthermore, no 
TABLE 3. Complication rate

\begin{tabular}{lccc}
\hline \multicolumn{1}{c}{ Variable } & $\begin{array}{c}\text { MIE } \\
(\mathbf{n}=\mathbf{9 2})\end{array}$ & $\begin{array}{c}\text { OE } \\
(\mathbf{n}=\mathbf{1 6 5})\end{array}$ & $\begin{array}{c}\boldsymbol{P} \\
\text { value }\end{array}$ \\
\hline $\begin{array}{l}\text { Recurrent laryngeal nerve } \\
\text { injury }\end{array}$ & $3(3)$ & $10(6)$ & .21 \\
Heart failure & & & \\
Stroke & $2(2)$ & $2(1)$ & .52 \\
Atrial fibrillation & $0(0)$ & $2(1)$ & .55 \\
Wound infection & $22(24)$ & $46(28)$ & .49 \\
Delayed gastric emptying* & $19(21)$ & $33(20)$ & .90 \\
Acute renal failure $\dagger$ & $17(18)$ & $31(19)$ & .91 \\
Deep venous thrombosis & $1(1)$ & $5(3)$ & .14 \\
Pulmonary embolism & $2(2)$ & $4(2)$ & .55 \\
Pulmonary complications & $3(3)$ & $4(2)$ & .57 \\
$\quad$ Pneumonia & $9(10)$ & $26(16)$ & .94 \\
Postoperative respiratory & $2(2)$ & $21(13)$ & .01 \\
$\quad$ failure & $7(8)$ & $14(8)$ & .78 \\
Reintubated & & & \\
Tracheotomy & $5(5)$ & $10(6)$ & .75 \\
Adverse events requiring & $2(2)$ & $4(2)$ & .75 \\
$\quad$ intervention & & & \\
Conduit torsion & & & \\
Anastomotic leak & $2(2)$ & $2(1)$ & .25 \\
Chylous leak & $5(5)$ & $7(4)$ & .65 \\
Esophageal stricture & $3(3)$ & $5(3)$ & .91 \\
Bleeding & $7(8)$ & $11(7)$ & .17 \\
\hline Dat presentan & $3(3)$ & $2(1)$ & .25 \\
\hline
\end{tabular}

Data presented as n (\%). MIE, Minimally invasive esophagectomy; $O E$, open esophagectomy. *Diagnosed by upper gastrointestinal series. $\dagger$ Defined by an increase in serum creatinine of $\geq 50 \%$.

operator-specific differences were present in the morbidity, mortality, or overall survival.

Similar to other minimally invasive procedures, the MIE was associated with a shorter length of stay, ICU admission rate, ICU length of stay, and lower estimated blood loss. ${ }^{18}$ The shorter operative times were not substantial and likely resulted from the advanced experience of the minimally invasive surgeons. In both cohorts, the operative times reported were prolonged owing in part to the limited data. The skin incision and skin closure times were not available for all patients. This required that the "operative time" include preparing, positioning, and moving the patient onto a stretcher with the appropriate monitoring for transportation to recovery. The procedures were performed in a teaching institution, which might have also played a factor.

MIE, in particular the transhiatal and transthoracic techniques, were also associated with significantly greater lymph node recovery. This might be attributable to the improved and magnified visualization that is afforded with the minimally invasive techniques. However, improved nodal clearance could also have been significantly affected by surgeon intent over time. Presently, there is a significant impetus on the part of both the surgeon and the pathologist to enhance nodal recovery rates. This, combined with a greater proportion of MIEs performed recently, might be sufficient to account for the differences in nodal recovery. When the

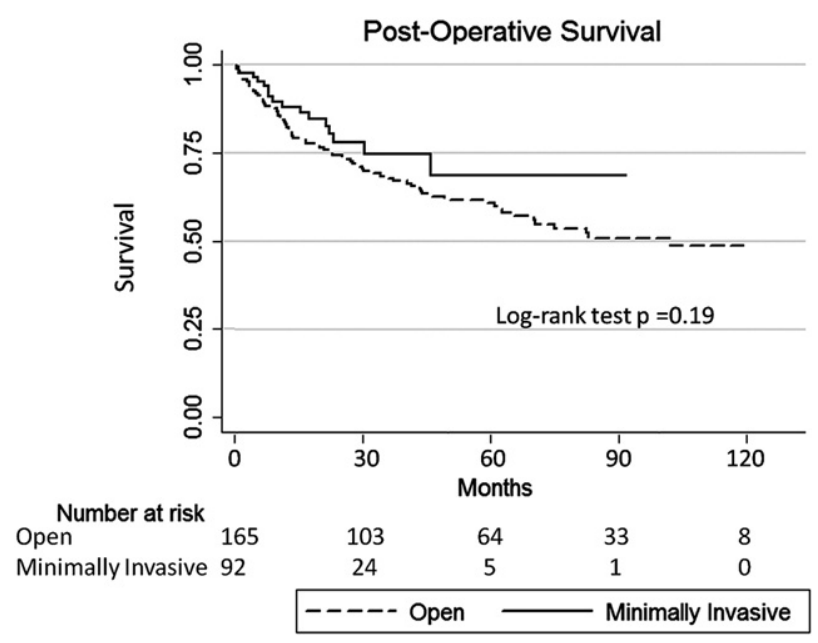

FIGURE 1. Overall survival for open and minimally invasive esophagectomy .

various techniques within each approach were compared, insignificant differences were found in the number of nodes harvested and their ranges overlapped. Therefore, we caution against concluding anything beyond comparable lymph node dissection.

In reporting adverse events, a distinction was made between anastomotic leaks as visualized on barium swallow testing or requiring a formal revision or washout versus leaks that contribute to wound infection but could be managed by opening the wound at the bedside. Only a portion of patients with wound infections had a leak defined by esophagography or that required operative evaluation or treatment. Our group has a very low threshold to open cervical incisions to rule out potential sources of infection, and these suspected leaks were included in the reporting of wound infections. As was the case with most other types of adverse events, no difference was found between the OE and MIE cohorts.

Consistent with other studies, the MIE group had a lower rate of postoperative pneumonia. ${ }^{18}$ The recurrent laryngeal nerve injury rate is thought to be underreported for all esophagectomies, because it is not the policy at our institution for postesophagectomy patients to routinely be examined by an ear, nose, and throat specialist. Patients are examined if clinical evidence is present to suggest vocal cord pathology, such as a failed speech and swallow examination or persistent hoarseness. Accordingly, instances of clinically insignificant recurrent laryngeal nerve injury might not have been detected.

The present study was limited by its retrospective, singleinstitution nature. Selection bias was lessened, and the learning curve with minimally invasive techniques was taken to account by using consecutive patients for a slightly more than 10-year span. The large sample size and similar composition of both cohorts strengthened the comparisons; 
however, it should be noted that the OE cohort had more patients with stage III disease. Therefore, any definitive conclusions regarding comparability might be limited to stage I and II disease. The subset of patients found to have stage 0 disease on final pathologic examination had a preoperative diagnosis of stage I, II, or III esophageal cancer. A select portion of these patients received combined induction chemoradiotherapy as outlined and had a complete pathologic response. The patients with preoperative stage I disease had T1a lesions according to preoperative endoscopic ultrasonography.

Given the 10-year period, there might be additional bias in terms of the management strategy over time and the development and refinement of the various MIE techniques during the earlier parts of our study. The percentage of MIEs increased in the second half of the study, and, as expected, the duration of follow-up for the MIE arm was shorter. The MIE cohort might have disproportionately benefited from any improvements in the postoperative management techniques that tend to occur with time. Any favorable results in the MIE group must be viewed through that prism. However, both open and minimally invasive procedures continue to be performed concurrently at our institution; thus, the bias might not be as great. This potential bias was further countered because postoperative management strategies specific to each approach (OE vs MIE) have changed little over time.

Admittedly, becoming facile with all 3 MIE techniques can be challenging. As our experience with MIE has grown, we have modified our technique to minimize the specific complications encountered. The IL MIE is now performed with a side-to-side completely stapled intrathoracic anastomosis to lessen the stricture rate. ${ }^{13}$ To minimize instances of chyle leak, we routinely ligate the thoracic duct in all procedures. To protect against possible future hernia formation, the stomach is anchored to the diaphragm during minimally invasive THE.

Without a prospective trial, we caution against using this or other retrospective studies to justify a paradigm shift away from $\mathrm{OE}$ as the standard of care for esophageal tumors. ${ }^{14,19}$ However, from our results, we can conclude that for early-stage esophageal cancer, a tailored minimally invasive technique can be offered to patients that maintains oncologic equivalence, including identical-to-improved lymph node dissection, with no differences in short- or long-term survival, and can be adopted by a single institution.

\section{References}

1. Turner GG. Excision of thoracic oesophagus for carcinoma with construction of an extra-thoracic gullet. Lancet. 1933;2:1315-6.

2. Lewis I. The surgical treatment of carcinoma of the esophagus, with special reference to a new operation for growth of the middle third. Br J Surg. 1946;34: 18-31.

3. McKeown KC. Total three-stage oesophagectomy for cancer of the oesophagus. Br J Surg. 1976;63:259-62.

4. Biere SS, Cuesta MA, van der Peet DL. Minimally invasive esophagectomy for cancer: a systematic review and metaanalysis. Minerva Chir. 2009;64:121-33.

5. Hulscher JB, Tijssen JG, Obertop H, Van Lanschot JJ. Transthoracic versus transhiatal resection for carcinoma of the esophagus: a meta-analysis. Ann Thorac Surg. 2011;72:306-13.

6. Hulscher JB, Van Snadick, de Boer AGEM, et al. Extended transthoracic resection compared with limited transhiatal resection for adenocarcinoma of the esophagus. N Engl J Med. 2002;347:1662-9.

7. Pennathur A, Zhang J, Haiquan C, Luketich JD. The "best operation" for esophageal cancer? Ann Thorac Surg. 2010;89:S2162-7.

8. Tinoco R, El-Kadre L, Tinoco A, Rios R, Sueth D, Pena F. Laparoscopic transhiatal esophagectomy: outcomes. Surg Endosc. 2007;21:1284-7.

9. Luketich JD, Alvelo-Rivera M, Buenaventura PO, Christie NA, McCaughan JS, Litle VR, et al. Minimally invasive esophagectomy: outcomes in 222 patients. Ann Surg. 2003;238:486-95.

10. Rice TW, Blackstone EH, Rusch VW. 7th Edition of the AJCC Cancer Staging Manual: esophagus and esophagogastric junction. Ann Surg Oncol. 2010;17: 1721-4.

11. American Thoracic Society; Infectious Diseases Society of America. Guidelines for the management of adults with hospital-acquired, ventilator-associated, and healthcare-associated pneumonia. Am J Respir Crit Care Med. 2005;171: 388-416.

12. Mathisen DJ, Grillo HC, Wilkens EW Jr, et al. Transthoracic esophagectomy: a safe approach to carcinoma of the esophagus. Ann Thorac Surg. 1988;45:137-43.

13. Gorenstein LA, Bessler M, Sonett JR. Intrathoracic linear stapled esophagogastric anastomosis: an alternative to the end to end anastomosis. Ann Thorac Surg. 2011;91:314-6.

14. Pennathur A, Awais O, Luketich JD. Technique of minimally invasive Ivor Lewis esophagectomy. Ann Thorac Surg. 2010;89:S2159-62.

15. Avital S, Zundel N, Szomstein S, Rosenthal R. Laparoscopic transhiatal esoph agectomy for esophageal cancer. Am J Surg. 2005;190:69-74.

16. Jamieson GG, Lamb PJ, Thompason SK. The role of lymphadenectomy in esophageal cancer. Ann Surg. 2009;250:206-9.

17. Braghetto I, Csendes A, Cardemil G, Burdiles P, Korn O, Valladres H. Open transthoracic or transhiatal esophagectomy versus minimally invasive esophagectomy in terms of morbidity, mortality and survival. Surg Endosc. 2006;20:1681-6.

18. Verhage RJJ, Hazebroek EJ, Boone J, Van Hillegersberg R. Minimally invasive surgery compared to open procedures in esophagectomy for cancer: a systemic review of the literature. Minerva Chir. 2009;64:135-46.

19. Luketich JD, Pennathur A, Catalano PJ, et al. Results of a phase II multicenter study of MIE (Eastern Cooperative Oncology Group Study E22020 abstract). $J$ Clin Oncol. 2009;27(Suppl):15S. 